Analysis of Axial Growth of Gold Indide by Indium Diffusing away from Solder Mounds and Creating the Gold to Gold-Indide Interface Angle Inside the Solder Mounds

W. J. Siekhaus, C. A. Hrousis

February 7, 2011 
This document was prepared as an account of work sponsored by an agency of the United States government. Neither the United States government nor Lawrence Livermore National Security, LLC, nor any of their employees makes any warranty, expressed or implied, or assumes any legal liability or responsibility for the accuracy, completeness, or usefulness of any information, apparatus, product, or process disclosed, or represents that its use would not infringe privately owned rights. Reference herein to any specific commercial product, process, or service by trade name, trademark, manufacturer, or otherwise does not necessarily constitute or imply its endorsement, recommendation, or favoring by the United States government or Lawrence Livermore National Security, LLC. The views and opinions of authors expressed herein do not necessarily state or reflect those of the United States government or Lawrence Livermore National Security, LLC, and shall not be used for advertising or product endorsement purposes.

This work performed under the auspices of the U.S. Department of Energy by Lawrence Livermore National Laboratory under Contract DE-AC52-07NA27344. 


\title{
Analysis of "Axial" Growth of Gold Indide by Indium Diffusing away from the Solder Mounds and Creating the Gold to Gold-Indide Interface Angle inside the Solder Mound. \\ Wigbert J. Siekhaus, Constantine Hrousis.
}

\begin{abstract}
.
The interfacial angle between gold and gold-indide in gold wires at the edge of PbSnIn $(37.5,37.5,25$. $)$ solder mounds has been deduced by measuring the reduction in gold-wire length caused by "axial" gold-indide growth along the surface of gold wires outside the solder mounds and comparing that growth to the known "radial" gold-indide growth inside the solder mound. That interfacial angle has been found to be, on average, thirty degrees independent of temperature for samples aged between ninety and sixty degrees for 3.9 months, but shows large scatter, from a minimum of fifteen degrees for fast axial gold-indide growth, to a maximum of ninety degrees for fast gold-indide growth.
\end{abstract}

\subsection{Introduction and Objective}

Gold bridge-wires on detonator-"headers" react with indium inside the solder mound to form gold indide, $\mathrm{AuIn}_{2}$, but also outside the solder mound due to indium diffusing away from the solder mound along the surface of the gold-wire. While the formation of gold indide inside the solder mound does not change the resistance and hence the performance of the bridge-wire, conversion of gold to gold-indide outside the solder mound does affect the performance of the bridge-wire: i.e. it locally increases the resistance at the interface between wire and solder mound and hence the energy deposition leading to earlier burst near the solder mounds. It is therefore important and the first objective of this effort to understand the kinetics of the transport of indium away from the solder mounds and its consequence: the shape of the interface between the gold wire and the solder mound as the gold-wire is being converted to gold-indide with increasing age.
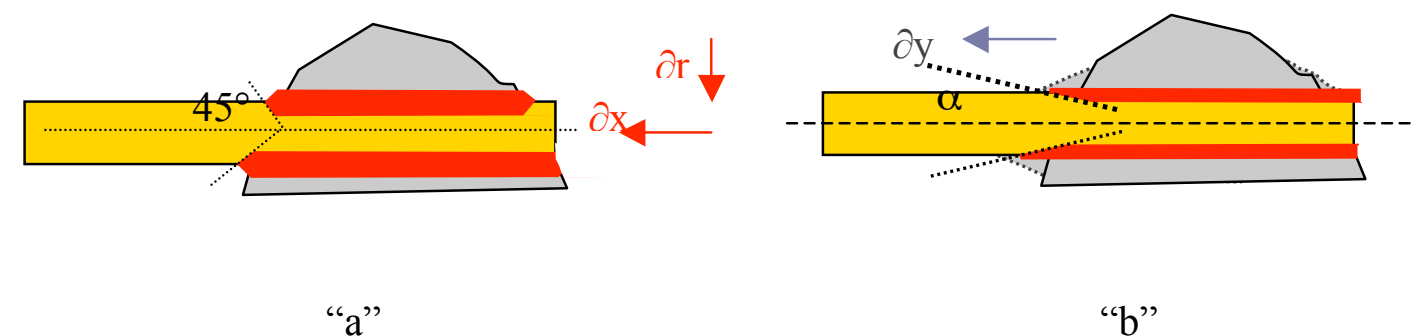

The sketch above illustrates the objective. In part "a" the indium in the solder mound does not move from the solder mound along the wire. Only the gold-indide (in red) grows in radial direction with the rate $\partial \mathrm{r} / \partial \mathrm{t}(\mu \mathrm{m} / \mathrm{month})$ and in the axial direction with the rate $\partial \mathrm{x} / \partial \mathrm{t}(\mu \mathrm{m} / \mathrm{month})$. Since the reaction rate is controlled solely by the rate at which the gold interface can convert itself to gold-indide, it follows that $\partial \mathrm{r} / \partial \mathrm{t}=\partial \mathrm{x} / \partial \mathrm{t}$, and one would have an interface shape as shown in "a". $\partial \mathrm{r} / \partial \mathrm{t}$, the linear reaction rate, is known and is equal to $1.4510^{-10}{ }^{*} \exp$-[7990/T]. If, however, as shown in "b", the solder diffuses along the wire with a rate $\partial y / \partial t$, an interface shape as in " $b$ " results. The objective is then to determine $\partial \mathrm{y} / \partial \mathrm{t}$, and hence the shape of the interface, see details in figure 1. 
As gold-indide grows inside the solder mounds, it pushes the gold wire away from the header's surface while the wire outsider the mound is being "held down" by the high explosive above it, see figure 1 . A second objective is to determine the change in height above the base as a function of temperature and time, since it determines the degree of curvature of the wire as it ages.

\subsection{Experimental Methods}

"Headers" with bridge-wires of $38.1 \mu \mathrm{m}$ (1.5 mil) diameter soldered with standard solder $(37.5 \% \mathrm{~Pb}, 37.5 \% \mathrm{Sn}, 35 \% \mathrm{In})$ were aged at elevated temperature in air in electrically heated laboratory ovens, five each at 40,60, 75, 85, and 90 centigrade. They were photographed in "top-view" and "side-view" at a range of magnifications (to show either the whole assembly at lower magnification or just the wire with the wire/solder-mound interfaces at higher magnification) before aging and after 3.9 month at temperature. Changes in wire dimensions were determined by optical microscopy calibrated with a length-standard. The following dimensions were measured (see figure 1.) in high-magnification images before and after aging:

1) The length of the bridge-wire between the solder mounds as seen in top view and in side view,

2) The height of the bridge-wire above the base of the header near the solder mounds,

3) The dimensions of the gold-indide as it protrudes from the aged solder mounds (where feasible).

\subsection{Results and Discussion}

Figure 2 shows top-view and side-view images of one header (F17) before and after aging for 3.9 months at 90 centigrade with the measured lengths and heights as described in section 1.2 above, and graphically shown in figure 1. The length in side-view has decreased by about $60 \mu \mathrm{m}$ after aging, and in top view by about $50 \mu \mathrm{m}$. The height of the wire above the base has increased substantially, i.e. the wire has been "pushed" up above the header's surface by the gold-indide growth inside the solder mound by about $36 \mu \mathrm{m}$ at the right solder mound, and by about $20 \mu \mathrm{m}$ at the left solder mound.

The length and height measurements of all headers were statistically analyzed. Figure 3 shows the frequency distribution of fresh gold wire lengths as seen in top- and side- view. These two lengths show a strong correlation with each other. The side-view mean length is approximately $5 \mu \mathrm{m}$ less than the top-view length, caused partially by the fact that the solder mound is spherical. Figure 4 compares the gold wire lengthdistribution of all fresh headers observed in top view with the distribution of the five headers exposed to $90^{\circ} \mathrm{C}$ for 3.9 month. The mean length (based on the error function distributions used here) has been shortened through axial growth of $\mathrm{AuIn}_{2}$ by (522.71$457.95)=64.76 \mu \mathrm{m}$.

Figure 5 compares the distribution of the average heights of the gold-wires of all fresh headers and of the 5 headers aged at $90^{\circ} \mathrm{C}$ for 3.9 month. The average height of the aged samples has increased by $(42.701-8.5252)=34.18 \mu \mathrm{m}$ based on the error function distributions used there. That is a little less than twice the increase in radius caused by the gold-indide formation. Since the original wire diameter is $38.1 \mu \mathrm{m}$, and $1 \mathrm{~cm}^{3}$ of gold 
produces $4.08 \mathrm{~cm}^{3}$ of gold indide ${ }^{1}$ the maximum diameter of the gold indide wire that can be produced from a gold wire of $38.1 \mu \mathrm{m}$ is $76.95 \mu \mathrm{m}$, hence the conversion of gold to gold-indide inside the mound should only push the wire upwards by at most the increase in radius corresponding to the increase in diameter, i.e. by $(76.95 / 2-38.1 / 2)=19.43 \mu \mathrm{m}$. $76.95 \mu \mathrm{m}$ is also consistent with the diameter of the "bullnose" protruding from the solder mound in the images in the first row of figure 2 ( see the values of the bullnose diameter above the gold wire [first image in top row] and below the gold wire [second image in top row]. Those images show at the ends of the wire near the wire mounds first a dark substance whose maximum diameter is close to $76.2 \mu \mathrm{m}$, followed by a light-colored protrusion of larger diameter. One might be tempted to assume that the observed larger average increase in wire height is caused by "gold-indide" formed inside the solder mound that has much less than theoretical density. That is, however, not what one sees in cross section images of aged solder mounds. Figure 6 shows a series of optical micrographs of cross-sections through solder mounds at increasing aging time at low temperature (about 35 Centigrade). Also shown are calculated ratios of the increase in gold-indide volume (=DeltaRvolume) over the corresponding decrease in gold-volume (Deltarvolume) calculated from these images. That ratio is not quite the theoretical ratio (4.08) but rather on the average 4.48, indicating a nine percent decrease from theoretical density. One must therefore conclude that the wire is pushed up by other mechanisms, most likely minimization of the work needed to distort the solder mound as gold-indide grows. The deformation of the surrounding lead and tin grains visible if one compares the second and third image in figure 5 with the first clearly indicates that there are substantial forces at work as the gold-indide grows. In summary: the observed change in height is twice as much as one could expect from the gold-indide formation, and I have no reasonable explanation for that, but it is clearly real.

The average change in lengths [(top-length change + side-length change)/2] and in heights [(left-side height change + right-side height change)/2] of the actual 5 samples aged at each temperature to their original dimensions (rather than the dimension change comparison of aged samples to the whole fresh dimension distributions as done in figures 4 and 5) was calculated for samples aged at 60, 75, 85 and 90 centigrade. Values of $\partial \mathrm{y} / \partial \mathrm{t}$ $=[$ (measured average changes in wire lengths $) / 2] / 3.9$ month have been plotted in an Arrhenius plot in figure 7 together with the change in radius $\partial \mathrm{r} / \partial \mathrm{t}$ (@ $\mathrm{t}=3.9$ month) expected from the linear model \{"measured average changes in wire lengths" is defined as ( "'measured average change in wire length in top-view" plus "measured average change in wire length in side-view") divided by two $\}$. The activation energy for $\partial \mathrm{y} / \partial \mathrm{t}$, i.e. indium diffusion along the wire's surface, is close to the activation energy for $\partial \mathrm{r} / \partial \mathrm{t}$, the rate of decrease of the gold wire's radius, while the pre-exponential factor is greater by about a factor two. Also plotted is the measured average change in height/3.9 month. A Average change in height is defined as average of ("change in height at left solder mound plus change in height at right solder mound) divided by two"\}. Those latter data are not expected to be linear on an Arrhenius plot, since the change in total radius $\mathrm{R}$ (of gold indide surrounding the gold wire, as seen in figure 6) does not change linearly with

\footnotetext{
${ }^{1}$ One mole of gold (197 g) of density $19.3 \mathrm{~g} / \mathrm{cm}^{3}$ (a volume of $10.21 \mathrm{~cm}^{3}$ ) produces one mole of gold indide $\left(\mathrm{AuIn}_{2}, 197+2 * 115=427 \mathrm{~g}\right.$ ) of density $10.266 \mathrm{~g} / \mathrm{cm}^{3}$ (a volume of $41.6 \mathrm{~cm}^{3}$ ), a change in volume by a factor 4.08 , a change in radius by a factor 2.02 .
} 
decrease in gold wire radius $\mathrm{r}$ - except approximately for very small decreases in $\mathrm{r}$ from its original value where $-2 \pi \mathrm{rdr} * 4.08=2 \pi \mathrm{rdR}$. At $35^{\circ} \mathrm{C} \partial \mathrm{r} / \partial(\mathrm{t}=3.9 \mathrm{month})$ is .306307 , and the rate of indium diffusion $\partial y / \partial(t=3.9$ month $)$ is .435177 . Hence the tangent of the angle $\alpha$ in the sketch in section 1.1 is .704 , and $\alpha$ is $35^{\circ}$. The rise in wire height above the header base is estimated to be $1.16 \mu \mathrm{m} / 3.9$ month at 35 Centigrade.

The statistical analysis of the all height- and length-data is shown in appendix I. The standard deviations for the averaged "length in top-view" and "length in side-view" listed in appendix I are used in appendix II to determine their combined standard deviation in wire length change $\partial \mathrm{y}$. The observed change in wire length $\partial \mathrm{y}$, as well as $(\partial \mathrm{y}$ plus one standard deviation, representing fast change in wire length due to fast axial $\mathrm{AuIn}_{2}$ growth) and ( $\partial \mathrm{y}$ minus one standard deviation, representing slow change in wire length) and in addition the change in wire radius $\partial \mathrm{r}$ calculated from the standard linear model are plotted as a function of inverse temperature in figure 8. All data are fitted with exponential functions that can be used to extrapolate to lower temperatures. The exponential functions fitted to average, fast, and slow " $\partial \mathrm{y}$, change in wire length" are used in figure 9 to plot the "AuIn ${ }_{2}$-gold - wire necking angle" $\alpha=\arctan (\partial \mathrm{r} / \partial \mathrm{y})$ as a function of inverse temperature. The average interfacial angle $\alpha=\arctan \left(\partial \mathrm{r} / \partial \mathrm{y}_{\text {Average }}\right)$ appears to be $\sim 30^{\circ}$ almost independent of temperature. There is, however, large variation in the axial growth and hence in the interfacial angle. As a consequence thereof, the angle at $35^{\circ} \mathrm{C}$ is $\sim 90^{\circ}$ for slow axial growth, and $\sim 15^{\circ}$ for fast growth. Figure 10 depicts the gold wire's average change in height above the header, as observed (together with \pm one standard deviation, see appendix I ), and as predicted by the linear model. The observed change in height at elevated temperatures is consistently higher by approximately a factor of two.

\section{Summary}

Objective 1. We determined the temperature-dependent angle $\alpha$ of the interface between gold and gold indide at the edge of the solder mound over a range of temperatures and, by extrapolation, at a temperature of $35^{\circ} \mathrm{C}$. The average angle $\alpha$ is $30^{\circ}$, almost independent of temperature. There is, however, a large spread in observed angles, so that at low temperature angle values between 15 and 90 degrees may occur.

Objective 2. We have determined the gold wire's change in height above the header's surface and it is approximately twice as large as would be expected from the change in wire diameter induced by gold-indide growth. There is no good explanation for that phenomenon. 


\subsection{Figures}

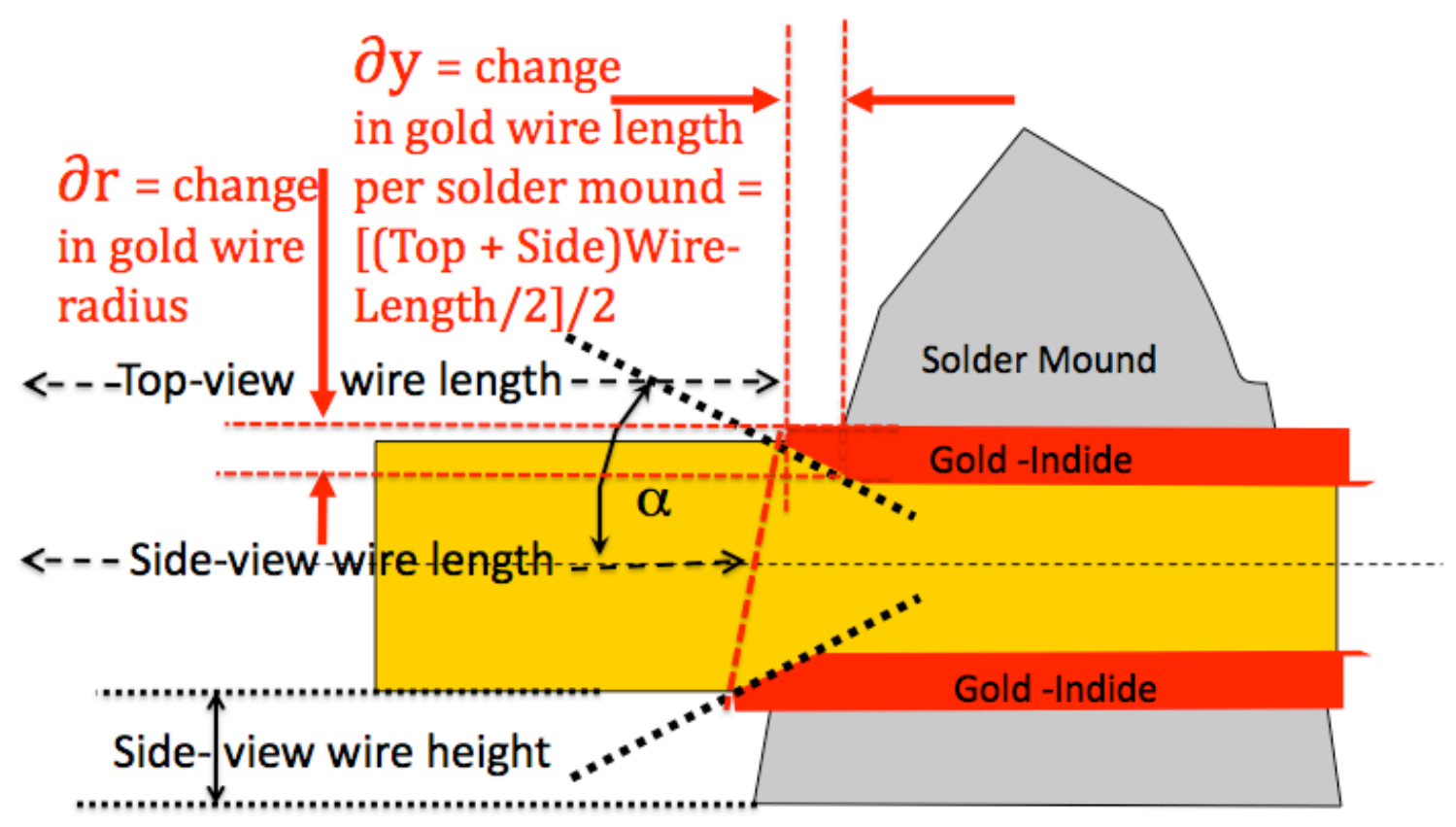

Figure 1. The interface between gold and gold-indide in an aged solder mound. Goldindide has grown beyond the edge of the solder mound by $\partial \mathrm{y}$, and the gold wire radius has been decreased by $\partial \mathrm{r}$, forming the interface angle $\mathrm{a}=\arctan (\partial \mathrm{r} / \partial \mathrm{y})$ between gold and gold-indide. 


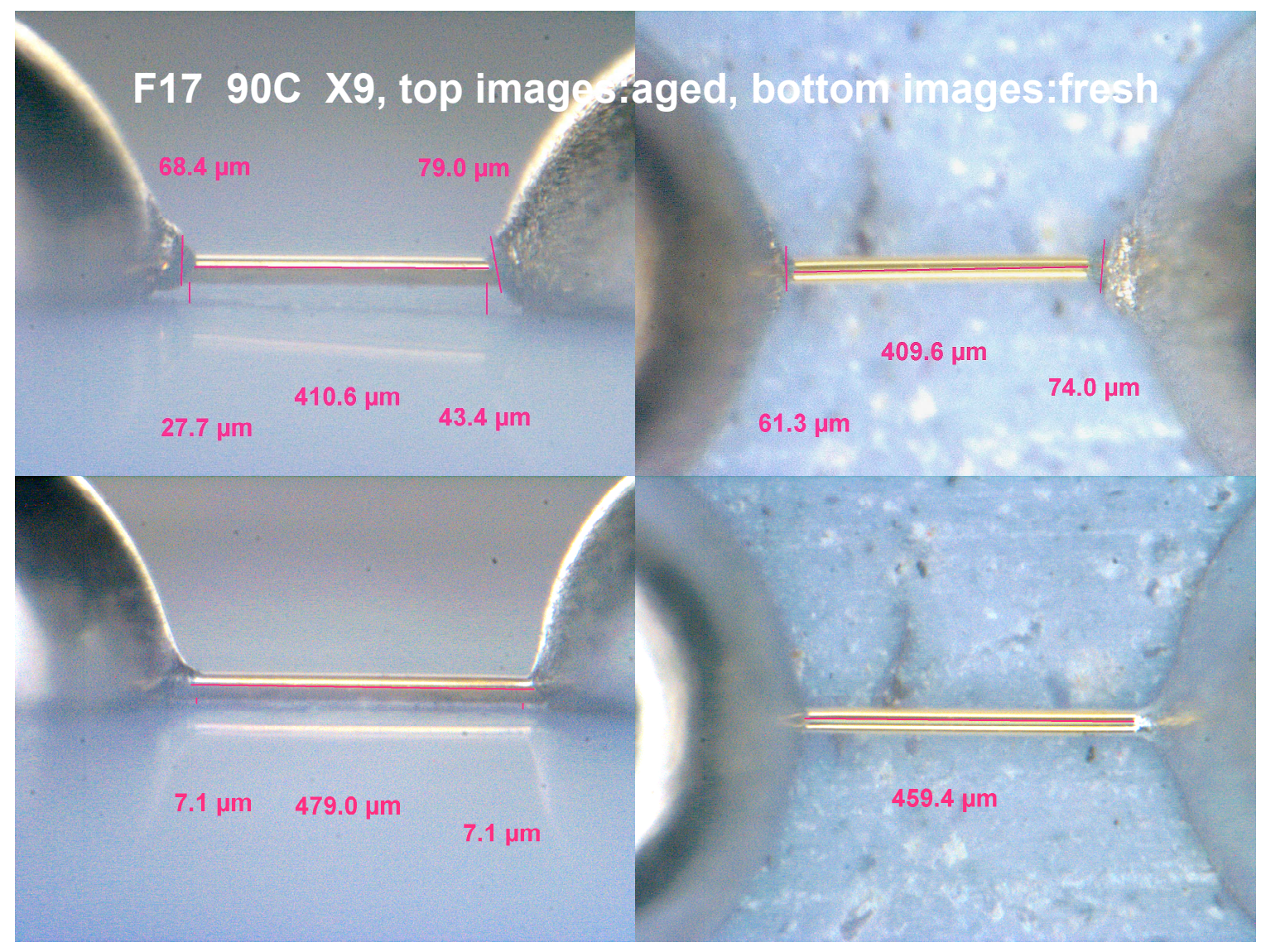

Figure 2. Optical micrographs of header F17 in side-view and in top-view after (top-row) and before (bottom row) exposure to $90^{\circ} \mathrm{C}$ for 3.9 month. The first (side-view) image in the top row shows the wire length as well as the diameters of the reaction product and the heights of the wire above the header base at the solder mounds. The second (top-view) image in the top row shows the length of the wire and the width of the reaction products at the solder mounds. The second row shows the same measurements before exposure to $90^{\circ} \mathrm{C}$. There are no reaction products there. 


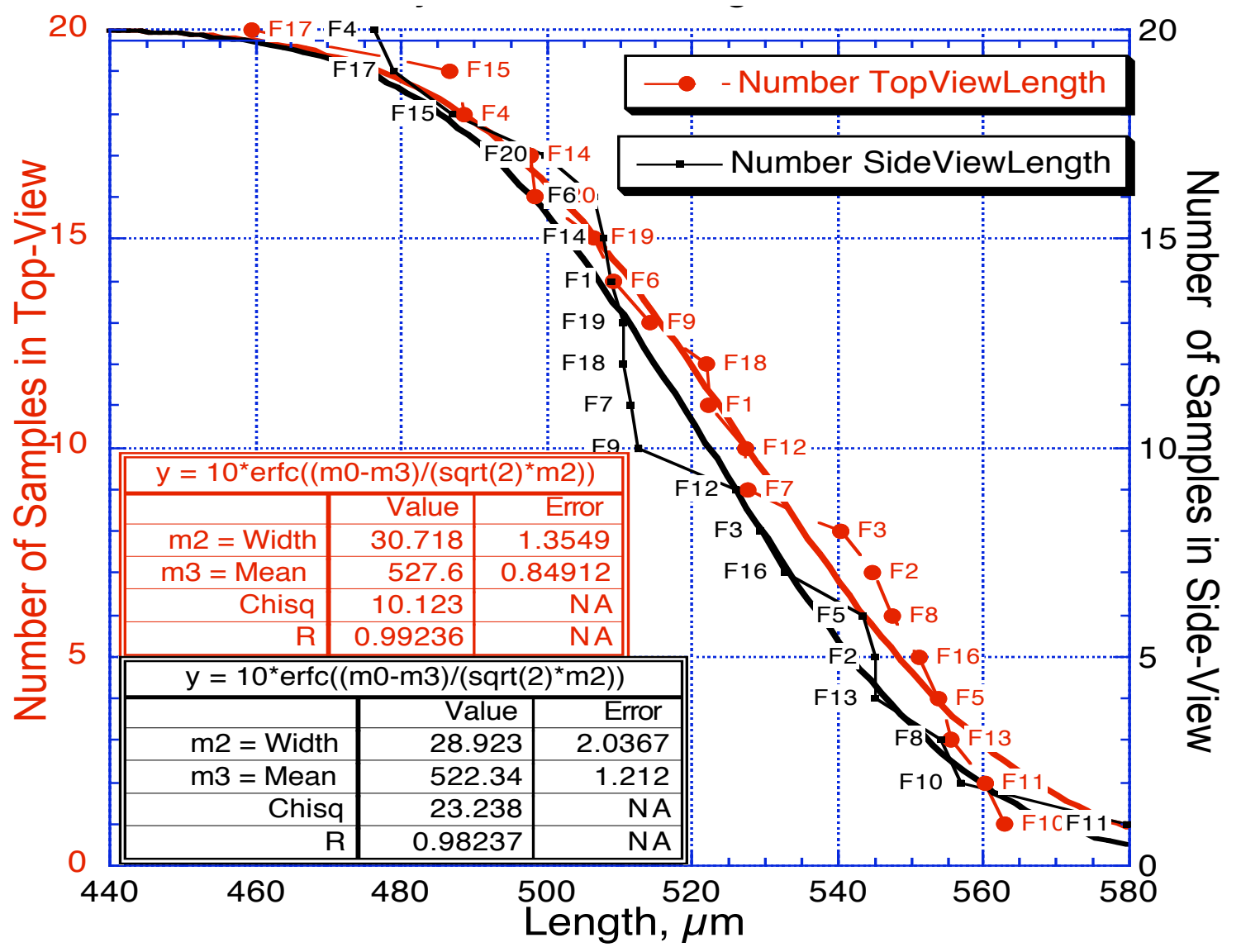

Figure 3. Top [red filled circles] - and side-view[black filled circles] length distribution of freshly soldered bridge-wires F1-F20. The mean length in side view is shorter by about $5 \mu \mathrm{m}$ because of the curvature of the hemispherical solder mounds. 


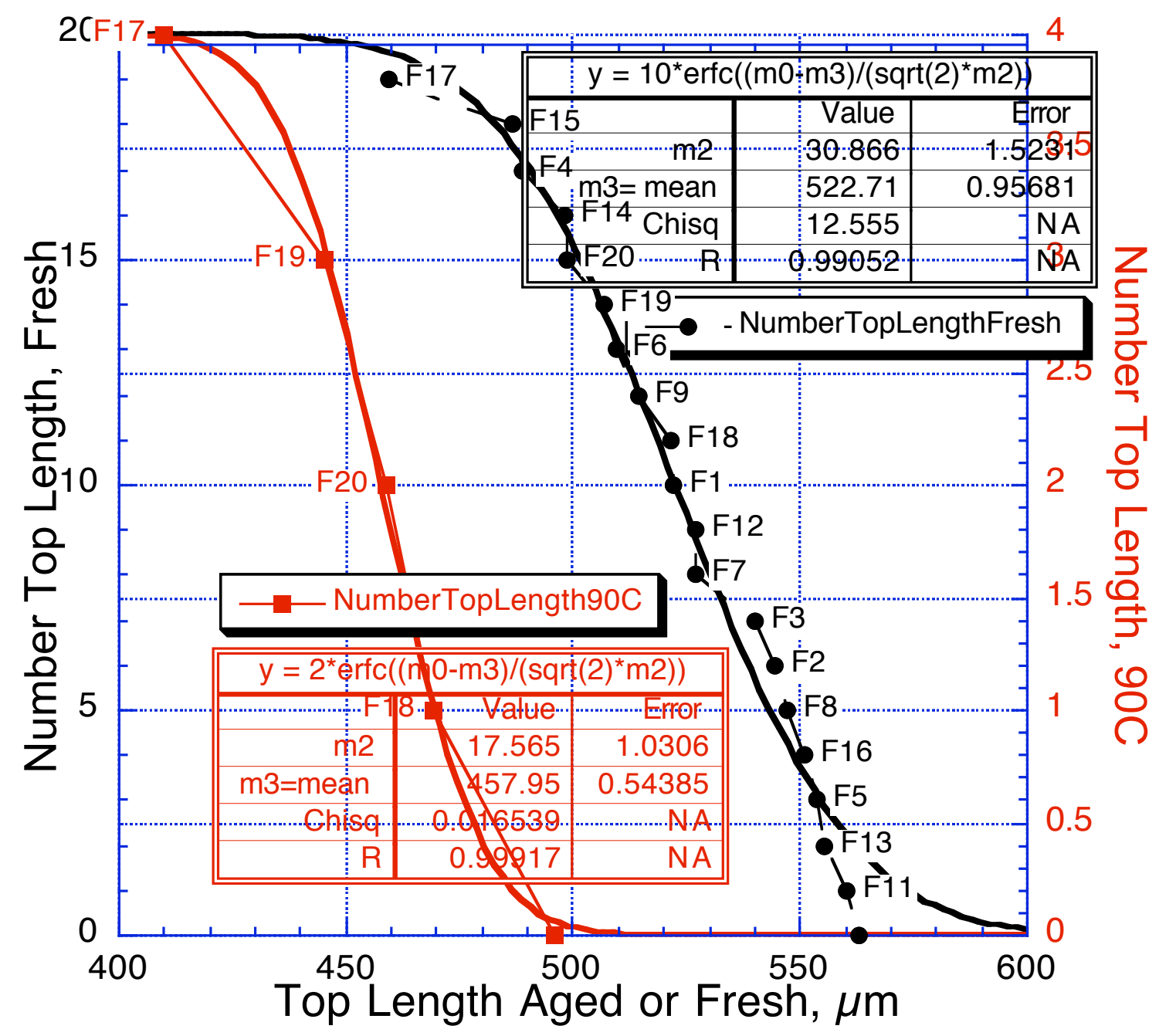

Figure 4. Comparison of the gold wire length observed in top view before [black filled circles] and after exposure to $90^{\circ} \mathrm{C}$ for 3.9 month [red filled circles]. The mean length (based on the error function distribution used here) has been shortened by (522.71$457.95)=64.76 \mu \mathrm{m}$. 


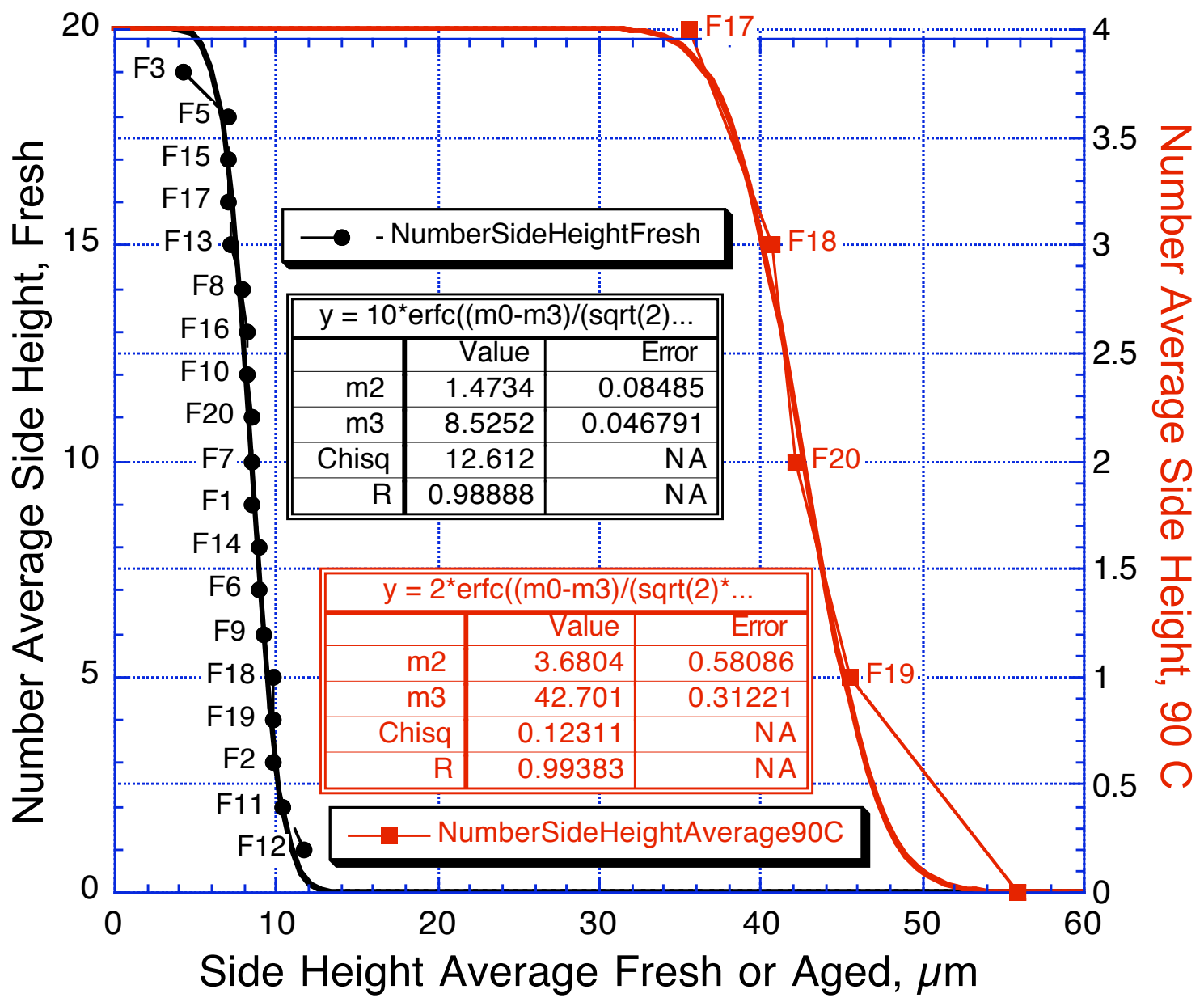

Fig. 5. Distributions of average height of the gold-wires of all fresh headers [black filled circles] and of the five headers aged at $90^{\circ} \mathrm{C}$ for 3.9 month [red filled circles]. The average height has increased by $(42.701-8.5252)=34.18 \mu \mathrm{m}$ based on the error function distributions used here. That is a little less than twice the increase in radius caused by the gold-indide formation. 


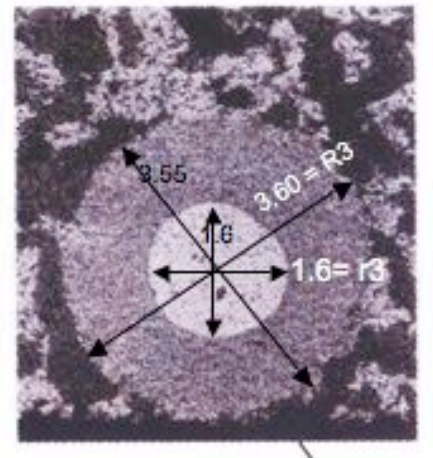

Del taRvoluee $3=R 2^{\wedge} 2-R 3^{\wedge} 2$ Deltarqolues $3=\mathbf{r} 3^{\wedge} \mathbf{z}-\mathbf{r} 2^{\wedge} 2$ Del taRrolues / Deltarqoiume 3 $=4.56984$

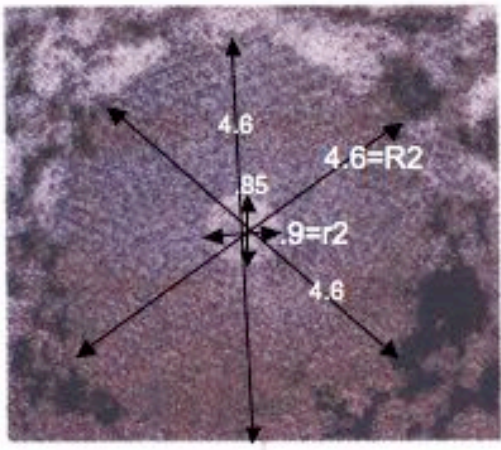

De1 taRrolune $Z=\mathbf{R} 1 \wedge Z-\mathbf{R} Z \wedge Z$

Deltarvoluee $2=r 2^{\wedge} 2$

DeltaRvolune2 / Deltarvolune? $=4.36571$

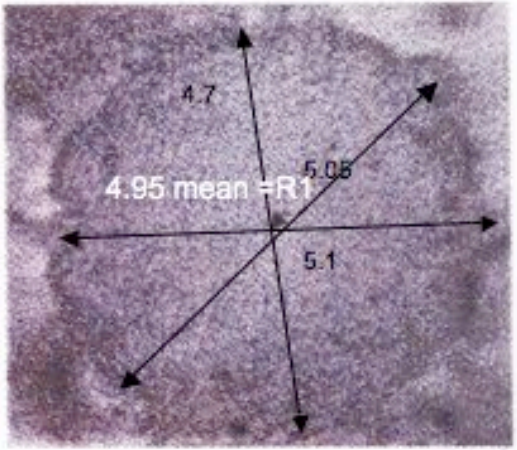

De1 taR rolume $1=\mathbf{R} 1 \wedge 2-\mathbf{R} \mathbf{3}^{\wedge} \mathbf{2}$

Deltarvolune1 $=r 3^{\wedge} 2$

DeltaRvolume1 / Deltarvolume1 $=4.50879$

Fig.6. A series of optical micrographs of cross-sections through solder mounds at increasing aging time at low temperature. Also shown are calculated ratios of the increase in gold-indide volume (=DeltaRvolume) over the corresponding decrease in gold-volume (Deltarvolume). That ratio is not quite the theoretical ratio (4.08) but rather 4.48, indicating a nine percent decrease from theoretical density. 


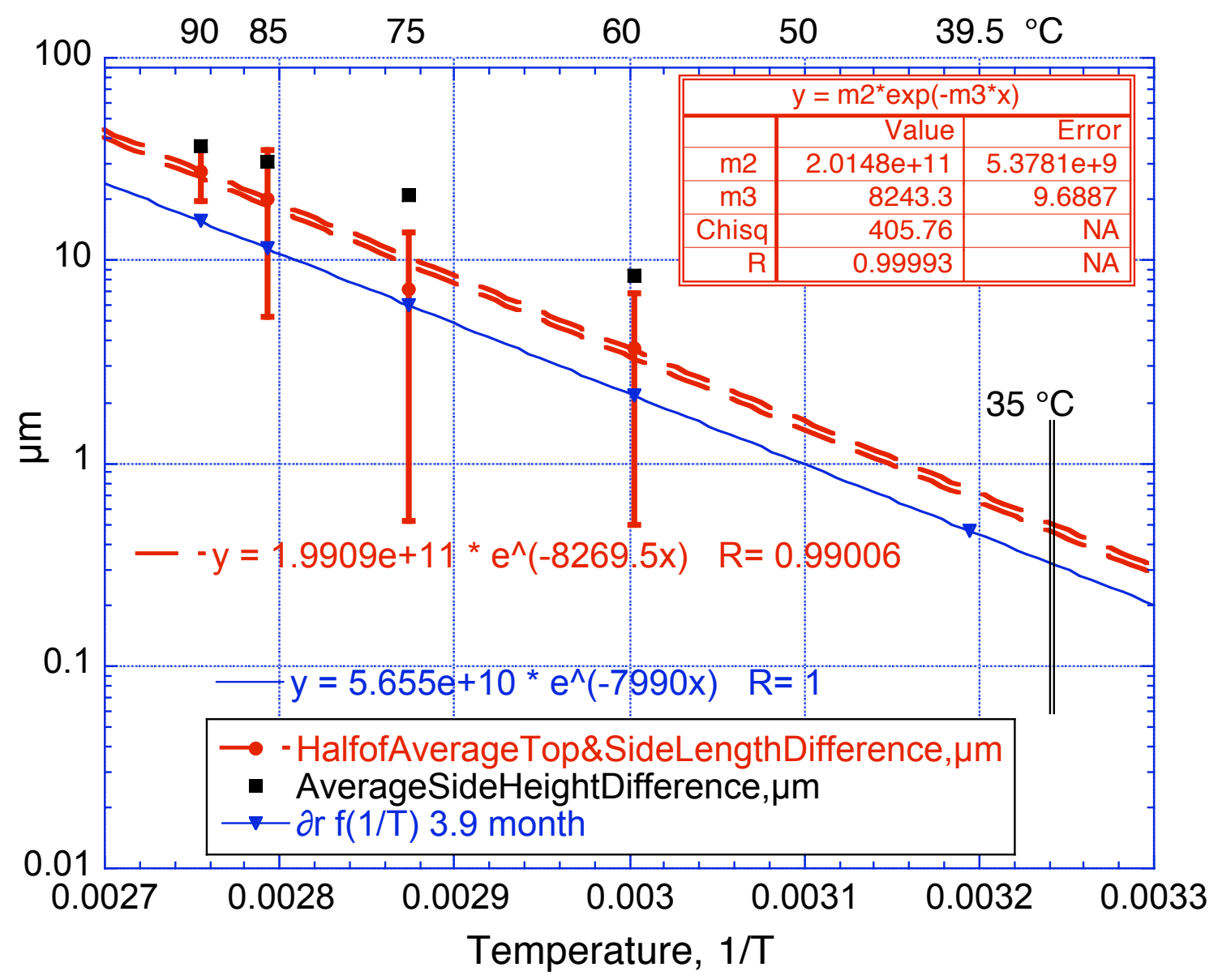

Figure 7. $\partial \mathrm{y} / \partial \mathrm{t}[\mathrm{red}$ circles $]=[($ measured average changes in wire lengths $) / 2] / @ 3.9$ month together with its error bars ( \pm one standard deviation, see appendix II) and the change in radius $\partial \mathrm{r} / \partial \mathrm{t}$ [blue triangles] (@ $\partial \mathrm{t}=3.9$ month) expected from the linear model, plotted versus $1 /{ }^{\circ} \mathrm{K}$ and versus the corresponding temperature in ${ }^{\circ} \mathrm{C}$. The fit parameters in the "red-lined-box" belong to the upper of the two dashed red lines. The datum at $75^{\circ} \mathrm{C}$ is ignored in that fit. The fit parameters written in red below the red and blue lines belong to the lower of the two dashed red lines. The datum at $75^{\circ} \mathrm{C}$ is included in that fit. Also plotted [in black squares] is the measured (height change/@3.9 month). 


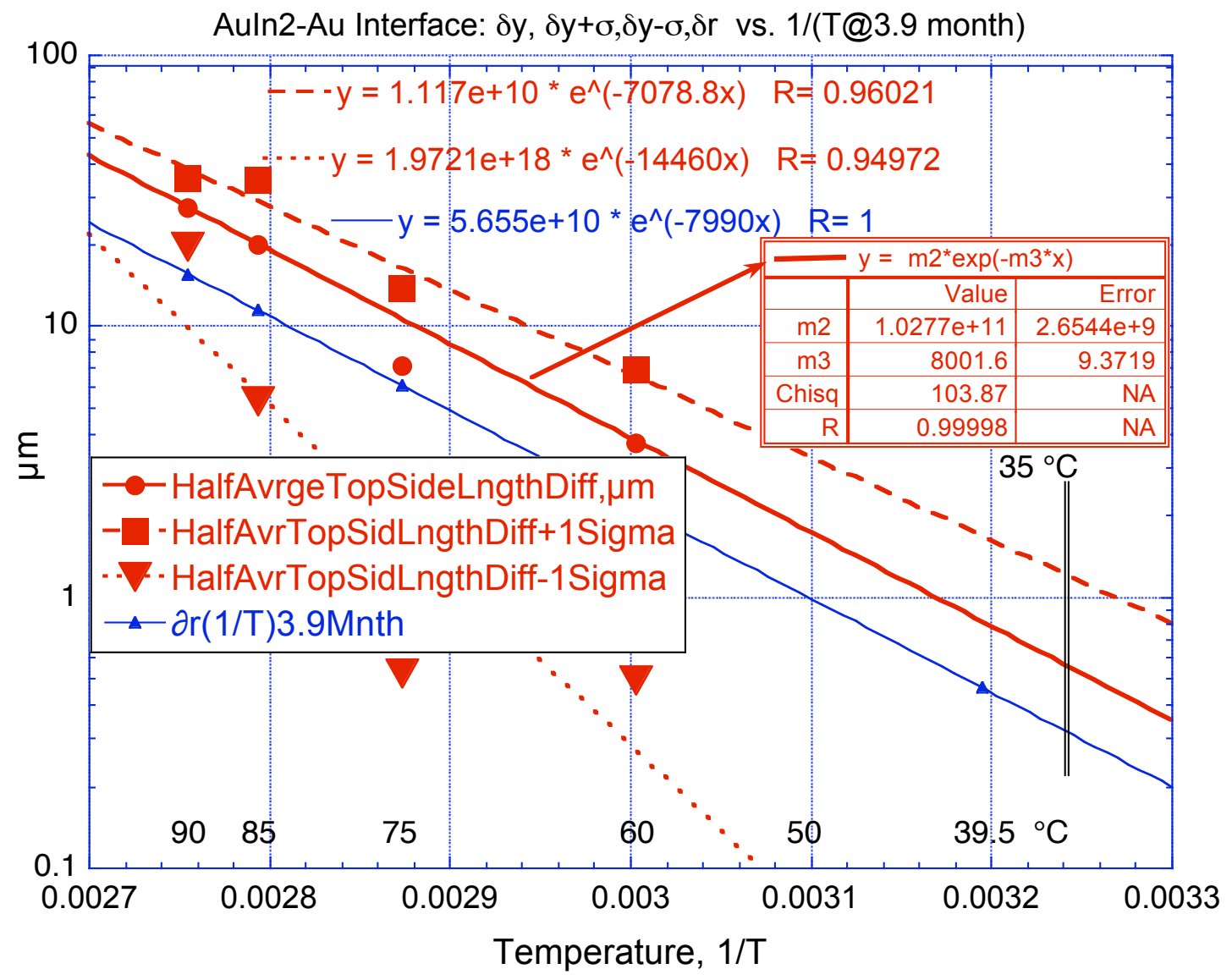

Figure 8. The observed change in wire length $\partial \mathrm{y}$ [red filled circles], as well as ( $\partial \mathrm{y}$ plus one standard deviation[red filled squares]) and ( $\partial \mathrm{y}$ minus one standard deviation[red filled triangles]) and in addition the change in wire radius $\partial \mathrm{r}$ calculated from the standard linear model [small blue triangles] plotted as a function of inverse temperature. The data are fitted with exponential functions. The standard linear model has an activation energy of $7990\left({ }^{\circ} \mathrm{K}\right)$ while the fit to $\partial \mathrm{y}\left(\right.$ done ignoring the point at $\left.75^{\circ} \mathrm{C}\right)$ has an almost identical activation energy of 8001.6 , but a pre-exponential factor about twice as large. 


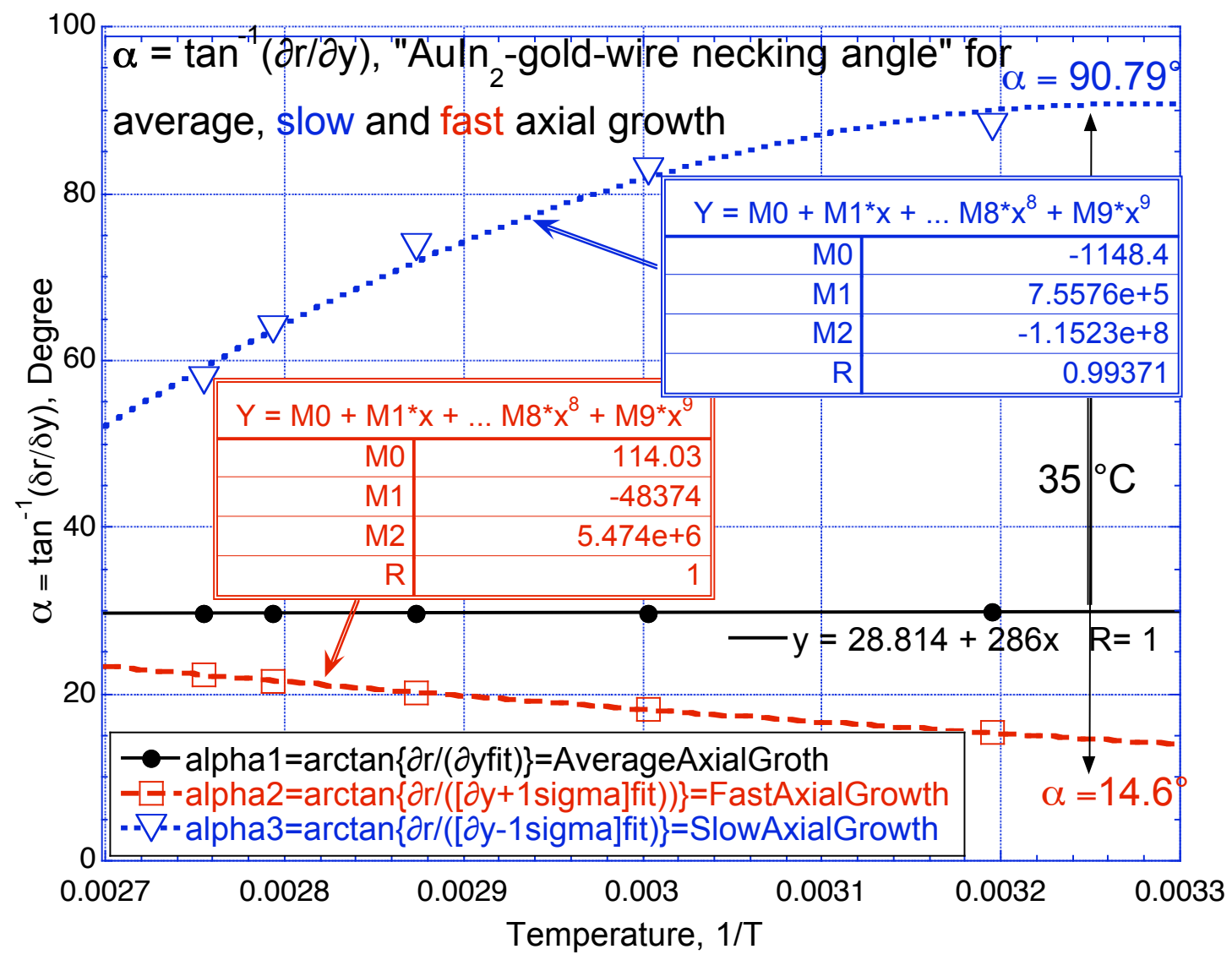

Figure 9. This graph plots interfacial angles $\alpha=\arctan (\partial \mathrm{r} / \partial \mathrm{y})$ calculated from the curves fitted to the experimental data shown in figure 8. The average interfacial angle $\alpha$ appears to be approximately $30^{\circ}$, almost independent of temperature (black circles). There is, however, large variation in the axial growth and hence in the interfacial angle. For slow axial growth the angle at $35^{\circ} \mathrm{C}$ is extrapolated to be $\sim 90^{\circ}$ (open blue triangles), for fast growth $\sim 15^{\circ}$ (open red squares). 


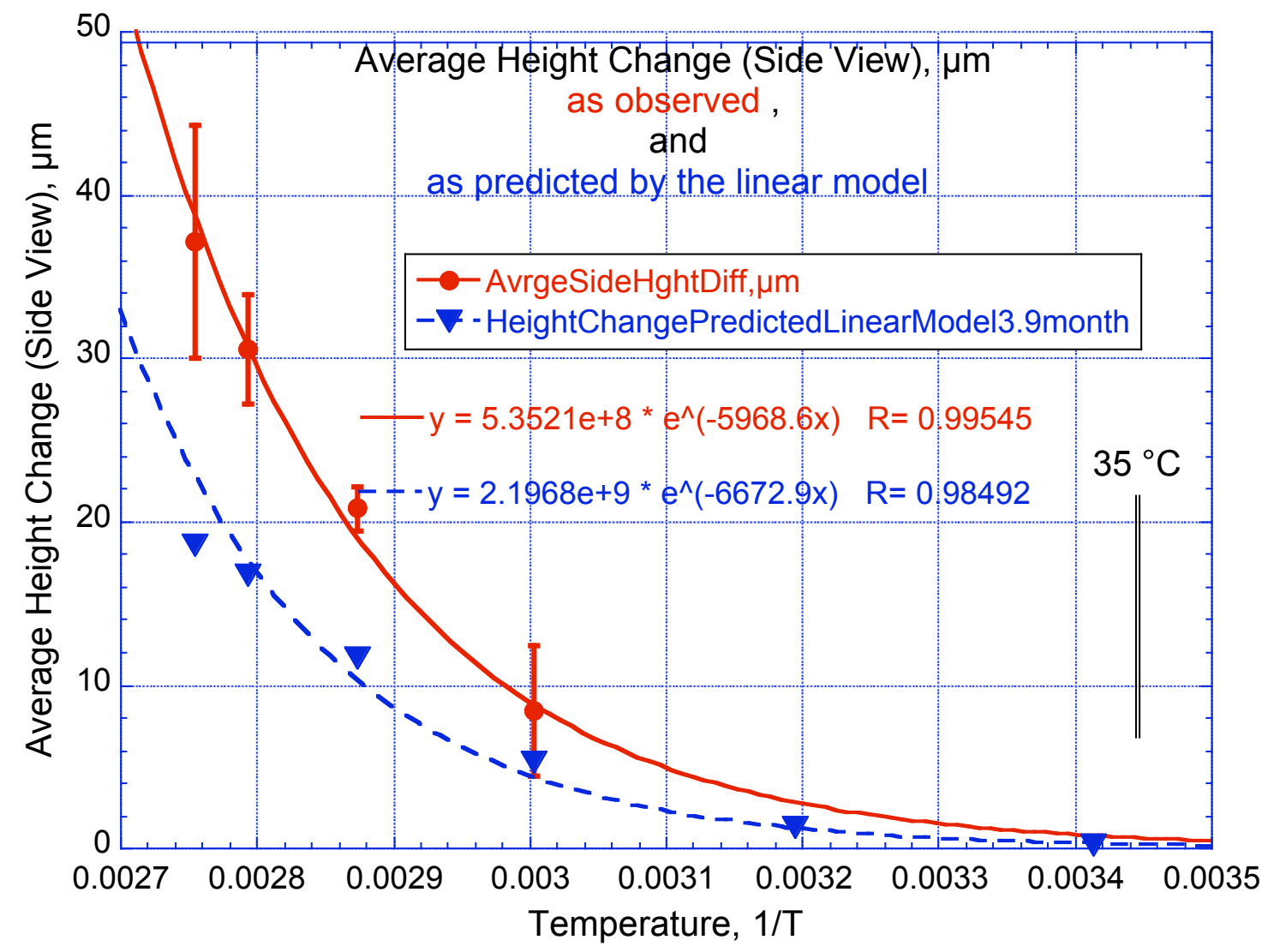

Figure 10. The gold wire's average change in height above the header, as observed ( red circles together with \pm one standard deviation, see appendix I ), and as predicted by the linear $\mathrm{AuIn}_{2}$ reaction model (blue triangles). 


\section{Appendix I.}

Statistical analysis of the "observed changed in height in side view", "observed change in length in top-view", "observed change in length in sideview", see figure 1 .

Taken out of the system 10_09_08,

Minimum 0

SideLengthDiff.Frsh $60^{\circ} \mathrm{C}$

Maximum 12.5

Sum 27.199982

Points 5

Mean 5.4399963

Median

6

RMS 6.9186692

Std Deviation 4.7794382

Variance $\quad 22.84303$

Std Error $\quad 2.1374297$

Skewness $\quad 0.40517221$

Kurtosis $\quad-0.9208364$

TopLengthDiff.Fresh $-60^{\circ} \mathrm{C}$

Minimum 5.5

Maximum $\quad 15.800018$

Sum 46.853363

Points 5

Mean 9.3706726

Median $\quad 8.0999756$

RMS 10.120307

Std Deviation 4.2736268

Variance $\quad 18.263886$

Std Error $\quad 1.911224$

Skewness $\quad 0.63590304$

Kurtosis $\quad-1.0571633$

\section{SideHeighDiff.Fresh- $60^{\circ} \mathrm{C}$}

Minimum 3.7999992

Maximum 13.9

Sum 42.199997

Points 5

Mean 8.4399994

Median $\quad 7.5499983$

RMS 9.150464

Std Deviation 3.9524362

Variance $\quad 15.621752$

Std Error $\quad 1.7675832$

Skewness $\quad 0.28635333$ 


\begin{tabular}{|c|c|}
\hline \multirow{2}{*}{\multicolumn{2}{|c|}{$\begin{array}{lr}\text { Kurtosis } & -1.1988889 \\
\text { SideLengthDiff.Frsh } 75^{\circ} \mathrm{C}\end{array}$}} \\
\hline & \\
\hline Minimum & 4.9000244 \\
\hline Maximum & 27.599976 \\
\hline \multicolumn{2}{|c|}{ Sum $\quad 62.800018$} \\
\hline \multicolumn{2}{|l|}{ Points 4} \\
\hline \multicolumn{2}{|c|}{ Mean 15.700005} \\
\hline Median & 15.150009 \\
\hline \multicolumn{2}{|c|}{ RMS 17.643833} \\
\hline \multicolumn{2}{|c|}{ Std Deviation 9.2962155} \\
\hline Variance & 86.419622 \\
\hline Std Error & 4.6481077 \\
\hline Skewness & 0.20335111 \\
\hline Kurtosis & -0.99694077 \\
\hline \multicolumn{2}{|c|}{ TopLengthDiff.Fresh $75^{\circ} \mathrm{C}$} \\
\hline Minimum & 0 \\
\hline Maximum & 22.299988 \\
\hline \multicolumn{2}{|c|}{ Sum $\quad 51.5$} \\
\hline \multicolumn{2}{|l|}{ Points 4} \\
\hline \multicolumn{2}{|c|}{ Mean 12.875} \\
\hline Median & 14.600006 \\
\hline \multicolumn{2}{|c|}{ RMS 15.242948} \\
\hline \multicolumn{2}{|c|}{ Std Deviation 9.422091} \\
\hline Variance & 88.775798 \\
\hline Std Error & 4.7110455 \\
\hline Skewness & -0.57833892 \\
\hline Kurtosis & -0.99765678 \\
\hline \multicolumn{2}{|c|}{ SideHeighDiff.Fresh- $75^{\circ} \mathrm{C}$} \\
\hline Minimum & 18.950001 \\
\hline Maximum & 21.999998 \\
\hline \multicolumn{2}{|c|}{ Sum $\quad 83.299997$} \\
\hline \multicolumn{2}{|c|}{ Points 4} \\
\hline Mean 20. & 999 \\
\hline Median & 21.174999 \\
\hline RMS 20 . & 443 \\
\hline Std Deviat & 1.3219294 \\
\hline Variance & 1.7474973 \\
\hline Std Error & 0.66096469 \\
\hline Skewness & -0.7960184 \\
\hline Kurtosis & -0.90781522 \\
\hline
\end{tabular}




\begin{tabular}{|c|c|}
\hline \multicolumn{2}{|c|}{ TopLengthDiff.Fresh $85^{\circ} \mathrm{C}$} \\
\hline Minimum & 5.2999878 \\
\hline Maximum & 63.899994 \\
\hline Sum 19 & Sum $\quad 195.10001$ \\
\hline Points 5 & \\
\hline Mean 39.020001 & 39.020001 \\
\hline Median & 36.900024 \\
\hline & 43.783082 \\
\hline \multicolumn{2}{|c|}{ Std Deviation 22.20354} \\
\hline Variance & 492.99721 \\
\hline Std Error & 9.9297252 \\
\hline Skewness & -0.51405192 \\
\hline Kurtosis & -0.79286517 \\
\hline \multicolumn{2}{|c|}{ SideLengthDiff.Frsh $85^{\circ}$} \\
\hline Minimum & 21.700012 \\
\hline Maximum & 64.5 \\
\hline \multicolumn{2}{|c|}{ Sum 207.80109} \\
\hline \multicolumn{2}{|l|}{ Points 5} \\
\hline \multicolumn{2}{|c|}{ Mean 41.560217} \\
\hline Median & 36.299988 \\
\hline \multicolumn{2}{|c|}{ RMS 45.111528} \\
\hline \multicolumn{2}{|c|}{ Std Deviation 19.61499} \\
\hline Variance & 384.74782 \\
\hline Std Error & 8.7720901 \\
\hline Skewness & 0.2229442 \\
\hline Kurtosis & -1.7112113 \\
\hline
\end{tabular}

\begin{tabular}{ll}
\multicolumn{2}{c}{ SideHeighDiff.Fresh- $85^{\circ} \mathrm{C}$} \\
Minimum & 27.6 \\
Maximum & 35.949997 \\
Sum 152.85 & \\
Points & 5 \\
Mean & 30.569999 \\
Median & 30.15 \\
RMS & 30.714483 \\
Std Deviation & 3.3268965 \\
Variance & 11.068241 \\
Std Error & 1.4878334 \\
Skewness & 0.86904925 \\
Kurtosis & -0.56927599
\end{tabular}




\title{
SideLengthDiff.Frsh $90^{\circ} \mathrm{C}$
}

\author{
Minimum 37.399994 \\ Maximum 71.100006 \\ Sum 291.89999 \\ Points 5 \\ Mean $58.379999 \quad 64,76$ by curve comparison \\ Median 60.699982 \\ RMS 59.607901 \\ Std Deviation 13.457229 \\ Variance $\quad 181.09701$ \\ Std Error $\quad 6.0182558$ \\ Skewness $\quad-0.71405393$ \\ Kurtosis $\quad-0.80551052$ \\ TopLengthDiff.Fresh $90^{\circ} \mathrm{C}$ \\ Minimum 39.5 \\ Maximum $\quad 61.300018$ \\ Sum 258.39996 \\ Points 5 \\ Mean 51.679993 \\ Median $\quad 52.599976$ \\ RMS 52.176195 \\ Std Deviation 8.0260237 \\ Variance $\quad 64.417056$ \\ Std Error $\quad 3.5893469$ \\ Skewness $\quad-0.47515105$ \\ Kurtosis $\quad-0.68501895$
}

AverageSideHeightDiff.FRESH90C, $\mu \mathrm{m}$
Minimum 30.149998
Maximum 48.25
Sum 185.7
Points 5
Mean 37.14
Median 36.300003
RMS 37.68229
Std Deviation 7.1217453
Variance $\quad 50.719257$
Std Error 3.1849413
Skewness $\quad 0.69631009$
Kurtosis $\quad-0.77287859$

34.175 by curve comparison 


\section{Appendix II.}

Calculation of the statistical deviation of the gold wire length based on data in appendix 1.

"standard Deviation Top+Side View Length Difference $90^{\circ} \mathrm{C} "$ $\sqrt{8.0260237^{2}+13.457229^{2}} / 2$

Standard Deviation Top+Side View Length Difference $90^{\circ} \mathrm{C}$ 7.83444

"Standard Deviation Top+Side View Length Difference $85^{\circ} \mathrm{C} "$ $\sqrt{19.61499^{2}+22.20354^{2}} / 2$

Standard Deviation Top + Side View Length Difference $85^{\circ} \mathrm{C}$ 14.8134

"standard Deviation Top+Side View Length Difference $75^{\circ} \mathrm{C}$ " $\sqrt{9.422091^{2}+9.2962155^{2}} / 2$

"Standard Deviation Top+Side View Length Difference $75^{\circ} \mathrm{C}$ " 6.61807

"Standard Deviation Top+Side View Length Difference $60^{\circ} \mathrm{C}$ " $\sqrt{4.7794382^{2}+4.2736268^{2}} / 2$

Standard Deviation Top+Side view Length Difference $60^{\circ} \mathrm{C}$ 3.20573 